\title{
Formative assessment in the translation classroom: Closing a feedback loop
}

\author{
Sofiya Nikolaeva ${ }^{1}$, Tetiana Korol $^{2}$ \\ ${ }^{1}$ Department of Foreign Languages Methodology Teaching and Information and Communication Technologies, Kyiv \\ National Linguistic University, Ukraine \\ ${ }^{2}$ Department of Ukrainian, Foreign Languages and Translation, Poltava University of Economics and Trade, Ukraine
}

\begin{tabular}{l} 
Article Info \\
\hline Article history: \\
Received Dec 6, 2020 \\
Revised Apr 18, 2021 \\
Accepted May 7, 2021 \\
\hline Keywords: \\
Corrective teacher feedback \\
Feedback response type \\
Self-reflection report \\
Teaching translation \\
Undergraduate translators
\end{tabular}

Corresponding Author:

Tetiana Korol

Department of Ukrainian, Foreign Languages and Translation

Poltava University of Economics and Trade

3 Koval Street, Poltava, 36014, Ukraine

Email: koroltatyanalq@gmail.com

\begin{abstract}
Feedback as the key component of formative assessment was studied extensively in the context of the acquisition of the foreign language productive skills by different categories of learners. Similarly, this paper aimed at the investigation of the optimal design of the feedback loop in teaching translation to undergraduate students. A mixed-methods research involved 40 sophomores of Poltava University of Economics and Trade and was conducted during 2018-2019 academic year. It examined the efficacy of four feedback response types from both quantitative and qualitative perspectives. Sample Group 1 had to study the received teacher feedback arbitrarily and consider it, while working on their further translations. Sample Group 2 was required to prepare a written free-form self-reflection report on the teacher feedback. Sample Group 3 was supposed to resubmit their corrected translations to the teacher. Finally, Sample Group 4 had to accompany their self-reflection reports with the corrected translations. The obtained data showed statistically significant difference between the acquired levels of the translation skills by the students of the four sample groups. The highest results were achieved due to the combination of self-reflection reports and translation correction. The questionnaire on the students' attitude to these practices proved the received statistical data.
\end{abstract}

This is an open access article under the CC BY-SA license.

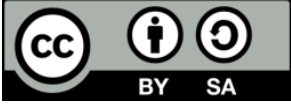

\section{INTRODUCTION}

Efficient assessment is considered one of the most challenging issues of modern didactics. It requires the development of the integrated network of the diversified assessment tools and techniques to comply with the specific educational aims and needs. They should ensure both reliable and objective information on the improvement of students' skills and training outcomes, provide the evidence of their professional development, monitor and promote learning and teaching systematically, and arrange university education process on a daily basis. The prevalence of one of the assessment functions listed above determines its type: 1) Summative assessment (assessment of learning); 2) Sustainable assessment (assessment as learning); 3) Formative assessment (assessment for learning) [1]-[4]. According to Kelly [5], formative assessment includes any grading, correcting or commenting of the students' product to provide them with the detailed feedback on their learning or particular task performance. It is directed to the improvement of their current training outcomes. This type of assessment stresses rather on quality than quantity, dynamics than 
statics, and causes necessary changes in teaching and learning processes. It distinguishes what the students already can do and what they need to learn and understand in order to make progress and perform better [6]. Formative assessment is put into effect with the help of either alternative, or conventional assessment tools and techniques accompanied with indispensable feedback.

In the context of undergraduate translators' training, formative assessment of their translation performance grows in its importance and appears to be quite promising [2], [7]-[13]. Since it promotes students' learning, delivers qualitative information on teaching efficiency, and reflects real-life peculiarities of translation service provision. A prevailing professional practice of correcting a target text (TT) according to reviewer's and editor's comments proves its authenticity as well. Moreover, formative assessment strongly supports social constructivist approach to teaching any specific translation type through and by doing. It gives the opportunity to involve students into the solution of real-life translation tasks and problems but reserves enough space for some kind of guidance and assistance. In case of formative assessment, they can naturally come in the form of a teacher corrective feedback. Different forms of feedback were primarily suggested and commonly used for teaching foreign language writing [14]-[20] and speaking [21], [22] to different categories of learners. To our mind, these findings can be righteously extended to teaching different types of translation as well. In its turn, the research of feedback application in undergraduate translators' instruction is focused on several aspects only. Južnič [8] studied the discrepancy and congruity between trainers' and trainees' feedback perception at Slovenian universities. Alfayyadh [9] compared feedback functions in American and Saudi Arabian educational environments. Neunzig and Tanqueiro [12], Yang, et al. [23] dealt with the peculiarities of feedback delivery in terms of computer-assisted translators' training. Washbourne [13] defined the features of the productive written feedback on students' translation performance according to dialogic and iterative approaches. Pietrzak [10] developed the design of group feedback exchange for the translation course. Lee [11] and Lim [24] singled out some feedback types in interpreters' training.

Despite the wide range of relevant studies, there is some space for further research. In our opinion, it should be concentrated on the development of the efficient feedback loop design to be applied in translation training and assessment at university level. Since this problem has not been studied yet from the perspective of the students' expected responses closing feedback loop and their impact upon learning and teaching outcomes. Therefore, the given paper aims at the development and verification of different feedback loop designs, which stipulate multiform types of the students' expected responses on the varied teacher feedback. In this context, the following research questions are addressed: 1) What type of expected response closes the feedback loop better and, as a result, promotes students' learning? 2) What is the students' attitude to the suggested and implemented feedback loop designs?

According to Barker and Pinard [25] iterative feedback is more helpful. Since it provides students with the opportunity to reflect on their performance and urges them to improve its quality. Moreover, any feedback serves its formative purpose only when it is actively used by the students in their further training [18], [20], [26]. In such a way, the assessment procedure converts into the two-channel dialogue loop [13], [27]. So, in order to close the feedback loop as the continuous productive process translator trainers should arrange iterative students' responses on the given feedback (correction, resubmission, and preparation of either oral or written response on feedback). That is why, the given research makes a novel attempt to compare the viability of different feedback loop designs and determine the most efficient one for undergraduate translators' training. Its findings will provide highly needed empirical evidence about the types of the students' expected responses, which promote their translation skills development, prove feedback importance in translation training and appeal contemporary students' attitudes. Consequently, this research will contribute to the advancement of the formative assessment practices used in the translation classroom.

\section{THEORETICAL BACKGROUND}

Feedback as the key component of formative assessment relates to the information provided by an agent to a learner concerning certain aspects of their performance [28]. Its main purpose is closing the gap between the current task performance and planned teaching and learning outcomes [7], [28]. To this end qualitative corrective feedback, represented in the form of either written verbal, or symbolic/coded comments, comes into play. Primarily, Lyster and Ranta [29] developed one of the most common corrective feedback taxonomy in modern applied linguistics. It is based on explicit/implicit and input-providing/outputprompting dimensions. This corrective feedback taxonomy comprises six categories, including explicit correction, recast, metalinguistic feedback/cue, elicitation, repetition and clarification request [29]-[31]. According to the objects they address, all corrective feedbacks can be divided into focused or intensive (concentrated on a particular aspect of the TT or even error type) and unfocused or extensive (devoted to the 
assessment and improvement of the general quality of the TT) [32], [33]. Unfocused corrective feedback is in agreement with the synthetic nature of translation performance and comprehensive assessment of its product in the training process. Later, Yang and Carless [27] represented feedback characteristics with the help of so called "feedback triangle". It contains three interrelated components or dimensions: 1) Cognitive (the content of the feedback); 2) Social-affective (the interpersonal negotiation of the feedback); 3) Structural (the organization of the feedback provision). Cognitive dimension reveals feedback actual content. In this vein, it outlines students' performance aspects; pinpoints applied and required translation strategies and techniques, specify possible ways for translation problem solution, and identify the directions of the feedback employment for the sake of the improvement of their translation skills. To our mind, it completely correlates with the corrective feedback classification by Lyster and Ranta [29] and incorporates its two aspects of explicitness implicitness and input-provision output-prompt. Social-affective dimension provides feedback interpersonal negotiation. It represents relevant roles (assessor/assesse) and their functions in training environment and involves some emotional background [34]. Structural dimension concerns the organization of feedback presentation in terms of timing, delivery channels, form, sequencing, expected responses as well as the ways of formative assessment management in general. It should be stressed here, that the last two dimensions are not reflected in the classification discussed above. Therefore, the combination of these two concepts allows us to develop more comprehensive feedback taxonomy for the formative assessment in the translation classroom. In this paper, we will consider the dimensions and aspects relevant to its focus only.

As for cognitive dimension, it entails feedbacks of different cognitive load. For instance, the explicit correction requires fewer students' efforts to be processed and employed than the clarification request. In this vein, we distinguish feedbacks of low, medium and high cognitive load. Feedbacks of low cognitive load usually supply students with clearly identified problem and ready-made or easy-to-use correction solutions. They include direct or explicit error/mistake correction by the assessor accompanied with relevant comment or explanation; direct or explicit error/mistake correction only, without any comments or explanations given; error/mistake locating in the TT with the help of highlighting, underlining or stressing, accompanied with several direct or explicit suggestions how to correct it. Feedbacks of medium cognitive load usually demand more thinking activity from the students. They guide their reflection and correction, but still grant some kind of free judgement and decision-making. Feedbacks of medium cognitive load comprise error/mistake locating in the TT with the help of highlighting, underlining or stressing, accompanied with its type identification (vocabulary use, syntactic structure application); error/mistake locating in the TT with the help of highlighting, underlining or stressing, accompanied with a leading question; out of text comment, listing all the errors/mistakes made and providing comprehensive instructions how to correct them. Feedbacks of high cognitive load usually engage students into full-scale reflection and correction process; since they provide some vague directions only. Consequently, feedbacks of high cognitive load involve error/mistake marking in the TT with either a question, exclamation mark or some emoji; out of text comment, listing all the mistakes and providing leading questions; standard general comments like: "Congratulations, you've managed to perform quite an acceptable translation. However, it still needs some review in terms of the source text sense reproduction" or "your translation would benefit a lot if you corrected some stylistic/grammar/spelling mistakes."

Regarding the objectives of our research, structural dimension of the feedback is primarily manifested in the type of the expected response. We range it from latent feedback studies without any external response, feedback studies and self-reflection report preparation, feedback-based correction of the TT segment or modification of the full TT to feedback-based correction of the TT preceded with a selfreflection report preparation. The influence of these different types of the expected response upon the efficiency and formative power of the assessment, in general, and feedback, in particular, in undergraduate translators' training is verified with the help of the research method.

\section{RESEARCH METHOD}

\subsection{Research design}

A mixed research design was employed in this survey. The descriptive qualitative research method shed the light on the multi-dimensional nature of the effective feedback in undergraduate translators' university training. The four types of students' expected response on the teacher written feedback were verified in terms of their impact on the students' translation skills development. The quantitative research method consisted in the processing and comparing of the dynamics of the undergraduate translators' grades received for the pre-test translation, 14 independently performed and submitted translations of English popular-science articles in Business Administration into Ukrainian during the spring term of 2018-2019 academic year and post-test (summative) translation. This method was also applied to conduct the statistical 
analysis of the obtained data with their further qualitative interpretation. To get more insights into the correlation of the students' learning outcomes and variable responses on the feedback, we used qualitative research in the form of students' questionnaire on their vision of the effective feedback arrangement and their attitude to the gained feedback experience.

\subsection{Research participants}

In total 40 second-year students, mostly females (32 female and 8 male respectively), aged from 17 to 19 years, majoring in Translation volunteered to take part in this research. It was conducted at Poltava University of Economics and Trade during the spring term of 2018-2019 academic year. The students have just started their first professionally oriented Translation Practice Course in the second term of their second bachelor year. The participants made up four sample groups (SG) of 10 students each. They studied in parallel groups and had taken the same basic foreign and native language courses before. The students' English language proficiency level ranged from B1+ to B2+ according to the CEFR scale. None of them was previously exposed to any translation training.

\subsection{Research instruments and procedures}

Taking into account limited contact hours for Translation Practice Course at Ukrainian universities and the volumes of learning materials and training activities, the students performed their translations out of class and submitted them to the teacher's correction and grading on a weekly basis. During the term, they had to prepare 15 translations. In general, we divided our research into five stages and described them in details in Table 1.

All the delivered translations during the whole research were graded and commented on by the same teacher according to 100 score analytical scale for the assessment of abstract translation modified from Shevelko [35] and given in Table 2. The selected criteria correlate with the essential skills of this type of translation and indicate their level of formation.

The presented criteria were implicitly reflected in the teacher feedbacks delivered to the students in terms of the errors/mistakes located, identified or corrected. The first translation was considered as a pre-test and its results were used as a benchmark for the further evaluation and interpretation of the received research data.

Table 1. Research structure

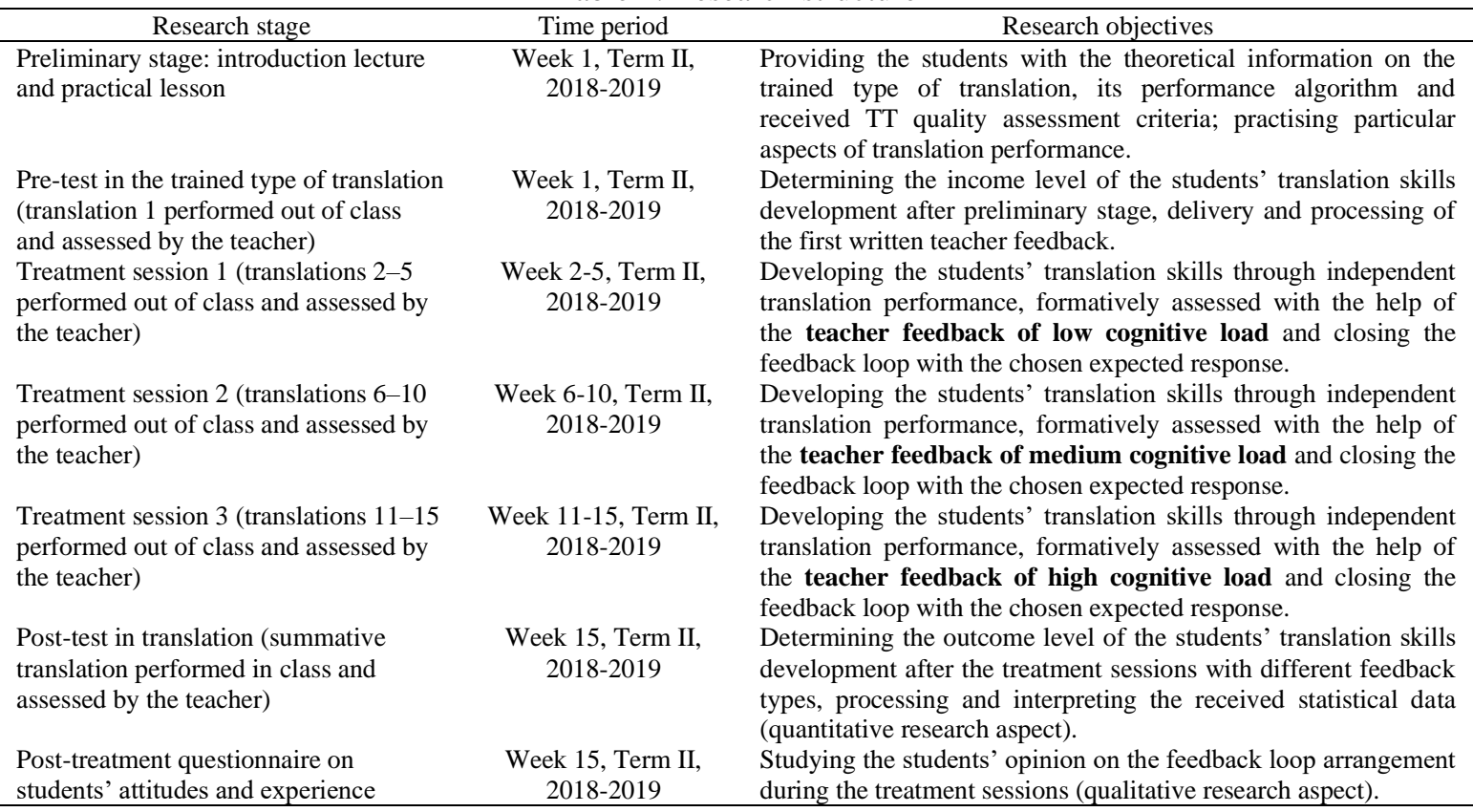


Table 2. Analytical scale for the assessment of abstract translation [35]

\begin{tabular}{ll}
\hline \multicolumn{1}{c}{ Criterion } & \multicolumn{1}{c}{ Examples of potential mistakes } \\
\hline $\begin{array}{l}\text { Adequate compression and } \\
\text { rendition of the source text } \\
\text { (ST) in the TT }\end{array}$ & $\begin{array}{l}\text { The lack of the main idea formulation; The lack of the secondary concepts } \\
\text { presentation; The lack of the author's conclusions rendition; The } \\
\text { misinterpretation of the idea status in the ST; The insufficient compression of the }\end{array}$ \\
$\begin{array}{l}\text { ST ideas and concepts. } \\
\text { The structure, cohesion and } \\
\text { coherence }\end{array}$ & $\begin{array}{l}\text { The lack of the relevant linking words and clichés in the TT; The misuse of the } \\
\text { linking words and clichés in the TT formulation; The ill-structured TT; Total in } \\
\text { cohesion or incoherence of the TT. }\end{array}$ \\
$\begin{array}{l}\text { Target language correctness } \\
\text { The wrong selection of the target language equivalents; Spelling, grammar and } \\
\text { TT presentation and layout }\end{array}$ & $\begin{array}{l}\text { syntactic errors; Stylistic mistakes; The occurrence of russianisms and literalisms. } \\
\text { The wrong presentation of the TT heading; Wrong margins, font, spacing, } \\
\text { indentation }\end{array}$ \\
\hline
\end{tabular}

The treatment stage comprised three consecutive sessions. They differed in the type of the teacher feedback provided to the students. During the first session, all SGs received the teacher feedback in the form of explicit error /mistake correction accompanied with the comments at first, then without them and, finally, they got several options to correct their translations (feedback of low cognitive load). The evolution of the teacher feedback formulation can be followed in such simple examples of correcting typical syntactic error in the ТT: "У статті розглядають ... (In Ukrainian we start a sentence with the preposition " $V$ ", but not “В”)," - "У статті розглядають ...". The correction options were mainly suggested in case of stylistic or transfer mistakes made by the students, when different formulations could be appropriate: "Use such clichés as: У статті проаналізовано..., Статтю присвячено вивченню проблеми ... Основну увагу автора зосереджено на ... to introduce the main problem discussed in the ST".

During the second session, the teacher preferred error / mistake locating and accompanied it with mistake/error type identification, or leading questions, or prompts, or correction guidelines (feedback of medium cognitive load). At this session, the teacher feedback for the correcting of the first abovementioned syntactic error looked like this: “B cmammi розглядають ... (Wrong preposition or WP)”. - “B cmammi розглядають ... (What preposition should we use at the beginning of the sentence?)”. As for the feedback on the second mistake presented above it looked like some local guidelines: "Study the sample list of the clichés introducing the main problem discussed in the ST and choose the one to be used in your translation".

Finally, yet importantly, the third session featured the teacher feedback of high cognitive load. It involved error / mistake marking with a question, exclamation mark or emoji, out of text mistake list with occasional leading questions or prompts or even standard text comment outlining translation strengths and weaknesses. At this treatment session, the teacher feedback on the syntactic error discussed above transformed into: “므 cmammi розглядають ...” or even just a passage of out of text comment like: “... Remember about the rules of preposition use at the beginning of the Ukrainian sentences. ..." There was an assumption, that the consistent increase of cognitive load in the teacher feedback would gradually engage the students into the practice of feedback processing and use, and foster their translation skills development. At the same time, the students of four SGs were required to process and respond on the feedback provided in different ways during the treatment sessions as shown in Table 3.

Table 3. The variation of the students' expected responses on the teacher feedback across the SGs

\begin{tabular}{cl}
\hline SG & \multicolumn{1}{c}{ Expected response on the teacher feedback } \\
\hline SG $1(\mathrm{n}=10)$ & $\begin{array}{l}\text { No explicit response is expected, the instruction is to study the given } \\
\text { feedback and take it into account, preparing the further translations }\end{array}$ \\
SG $2(\mathrm{n}=10)$ & $\begin{array}{l}\text { The preparation and submission of the written free-form self-reflection } \\
\text { report on the received feedback is required }\end{array}$ \\
SG $3(\mathrm{n}=10)$ & $\begin{array}{l}\text { The resubmission of the corrected translation version on the basis of the } \\
\text { received feedback is required } \\
\text { The preparation of the written free-form self-reflection report on the received } \\
\text { feedback and the resubmission of the corrected translations are required }\end{array}$ \\
SG $4(\mathrm{n}=10)$ &
\end{tabular}

Finally, the students of all SGs performed the post-test translation. The comparison of the pre-test and post-test results was aimed at the evaluation of the efficiency of the developed feedback loop designs for teaching translation to the second-year students. The given research was completed with the questionnaire on the trainees' attitude to the feedback and its application. It contained 10 open-end questions and was held online with the help of Google Forms. 


\section{RESULTS AND DISCUSSION}

For the consideration of the results of the assessment of the students' translations received after the introduction class at the pre-test stage see Table 4. Predictably, the students of all SGs produced abstract translations of insufficient quality in pre-test. Their performance scores fluctuated from minimum 38.3 (SG 4) to the maximum of 40.7 (SG 1 and 2), indicating the need for the further training and practising. The students' abstract translations obtained low scores according to all the assessment criteria. It is worth noting that the students of SG 4 demonstrated the lowest results compared to the others. The analysis of the intermediate assessment data of the performance of abstract translation 5 revealed sufficient improvement in terms of the students' skills to comply with formatting and layout requirements: maximum 10 out of 10 (SG 4). In addition, the students of SG 4 gained the highest increase indices according to all the assessment criteria with the highest total score of 55.0. While the students of SG 1 made the lowest progress (44.2 compared to the income score of 40.7). The analysis of the scores earned by the representatives of SG 1 during the conducted research supports the idea of the students' obligatory active participation and engagement into the feedback process. Feedback is not efficient if the student does not respond it, if followup actions are not taken [27]. The analysis of the scores of intermediate abstract translation 10 completely supports the provisions presented above. Higher cognitive pressure, generated by the teacher feedback, promoted better learning outcomes of the students of SG 2-4, who had to participate in closing the feedback loop through either self-reflection report, corrected resubmitted translation or both. According to the received data, in 10-week period only the students of SG 4 achieved minimum required score of 70.2. Finally, the post-test results proved higher efficacy of the students' active response on the teacher feedback: 89.5 - SG 4 (7 students got excellent marks out of 10); 83.8 - SG 3 (2 students got excellent marks out of 10); 78.1 - SG 2 (good marks only); 73.7 - SG 1 (5 good marks out of 10). The dynamics of the increase rate is presented in Table 5.

Table 4. The mean values of the students' translation assessment results across the research stages and SGs

\begin{tabular}{|c|c|c|c|c|c|}
\hline \multirow{2}{*}{$\begin{array}{c}\text { SG } \\
\text { index }\end{array}$} & \multicolumn{4}{|c|}{ Scores received according to certain assessment criteria } & \multirow{2}{*}{$\begin{array}{l}\text { Total } \\
\text { score }\end{array}$} \\
\hline & Content rendition & TT Structure and cohesion & Target language correctness & TT Presentation and layout & \\
\hline & 30 & 30 & 30 & 10 & 100 \\
\hline \multicolumn{6}{|c|}{ Pre-treatment results of abstract translation assessment } \\
\hline SG 1 & 12.8 & 10.8 & 11.8 & 5.3 & 40.7 \\
\hline SG 2 & 12.4 & 10.6 & 11.9 & 5.8 & 40.7 \\
\hline SG 3 & 12.2 & 10.8 & 11.2 & 5.3 & 39.5 \\
\hline SG 4 & 12.1 & 9.6 & 11.4 & 5.2 & 38.3 \\
\hline \multicolumn{6}{|c|}{ Intermediate treatment session 1 results (Abstract translation 5) } \\
\hline SG 1 & 13.3 & 12.2 & 12.9 & 5.8 & 44.2 \\
\hline SG 2 & 14.1 & 12.9 & 15.6 & 8.1 & 50.7 \\
\hline SG 3 & 16.6 & 14.9 & 16.0 & 8.0 & 55.5 \\
\hline SG 4 & 15.4 & 13.4 & 16.2 & 10.0 & 55.0 \\
\hline \multicolumn{6}{|c|}{ Intermediate treatment session 2 results (Abstract translation 10) } \\
\hline SG 1 & 17.0 & 15.6 & 17.0 & 8.8 & 58.4 \\
\hline SG 2 & 17.6 & 17.1 & 18.3 & 8.9 & 61.9 \\
\hline SG 3 & 19.2 & 17.5 & 20.1 & 9.9 & 66.7 \\
\hline SG 4 & 20.5 & 18.7 & 21.0 & 10.0 & 70.2 \\
\hline \multicolumn{6}{|c|}{ Post-treatment results of abstract translation assessment } \\
\hline SG 1 & 21.6 & 20.6 & 21.9 & 9.6 & 73.7 \\
\hline SG 2 & 22.3 & 22.7 & 23.3 & 9.8 & 78.1 \\
\hline SG 3 & 24.4 & 23.5 & 25.9 & 10.0 & 83.8 \\
\hline SG 4 & 27.3 & 25.4 & 26.8 & 10.0 & 89.5 \\
\hline
\end{tabular}

Table 5. The dynamics of the mean values increase across the research

\begin{tabular}{ccccccccccccc}
\hline Criteria & \multicolumn{4}{c}{ SG 1 } & \multicolumn{4}{c}{ SG 2 } & \multicolumn{3}{c}{ SG 3 } & \multicolumn{2}{c}{ SG 4 } \\
& Pre & Post & Increase & Pre & Post & Increase & Pre & Post & Increase & Pre & Post & Increase \\
\hline Content rendition & 12.8 & 21.6 & 8.8 & 12.4 & 22.3 & 9.9 & 12.2 & 24.4 & 12.2 & 12.1 & 27.3 & 15.2 \\
TT structure and cohesion & 10.8 & 20.6 & 9.8 & 10.6 & 22.7 & 12.1 & 10.8 & 23.5 & 12.7 & 9.6 & 25.4 & 15.8 \\
Target language correctness & 11.8 & 21.9 & 10.1 & 11.9 & 23.3 & 11.4 & 11.2 & 25.9 & 14.7 & 11.4 & 26.8 & 15.4 \\
TT presentation and layout & 5.3 & 9.6 & 4.3 & 5.8 & 9.8 & 4.0 & 5.3 & 10.0 & 4.7 & 5.2 & 10.0 & 4.8 \\
Total & 40.7 & 73.7 & 33.0 & 40.7 & 78.1 & 37.4 & 39.5 & 83.8 & 44.3 & 38.3 & 89.5 & 51.2 \\
\hline
\end{tabular}

The significance of the differences between the results of abstract translation performance by the students of the involved SGs was checked and proved statistically with the help of H-criterion KruskalWallis and Median Test calculated with the use of software Statistica 10.0. The received H value of 24.75 
and $\mathrm{p}<0.05$ allowed us to accept the alternative hypothesis about the existence of non-random differences between the average scores obtained by the students of the four SGs.

On this basis, we can conclude that properly designed feedback loop can serve efficiently as both monitoring and learning tool in teaching such a complicated cognitive and speech activity as translation. The received research results support the ideas by Barker \& Pinard [33] and Wasbourne [13] that any efficient feedback loop should start with the teacher comment and finish with the student's relevant response. We are strongly convinced that passive reception of the teacher feedback is inappropriate for the initial stages of translation training, because junior students mainly lack proper self-regulation skills. Consequently, they should be directed by the teacher to analyse the received feedback first with the help of some specifically developed tools, e.g., free-form or even guided self-reflection reports. This activity will ensure the deep studies of the teacher feedback, encourage translation problem formulation and direct the search for their solutions, arrange current translation reviewing and editing, develop some kind of feedforward strategies to fix or prevent relevant problems in the future and improve students' self-reflection, self-correction, selfassessment and self-regulation skills.

If we have a look at the self-reflection reports produced by the students of SG 2, we will notice that they are not always comprehensive enough contrasted to the ones presented by the representatives of SG 4 . At the same time, resubmitted translations by SG 3 still contained some previously commented or corrected by the teacher errors and mistakes. This can be explained by incomplete analysis of the teacher feedback content by the students if it is not verbalized or used in some intermediary activity. The translations revised and resubmitted by the students of SG 4 qualitatively differed from their previous versions, often being reformulated and corrected completely, but not in segments only. Iterative character of such feedback loop design combined with gradual cognitive load growth in the teacher feedbacks will foster the concurrent and efficient development of students' translation, cognitive and self-assessment skills. These results correspond to the conclusions reached by Washbourne [13] as for the need in versatile teacher feedback delivered in the translation classroom.

The received quantitative data were complemented with the students' questionnaire responses. All the students appreciated the idea of receiving the teacher written feedback on their translations individually that completely correlates with the similar results received previously by Južnič [8] and Lee [11]. However, the students of SG 1 valued feedback as the fact of their teacher's attention only, while the students of SG 2 treated it as the incentive to analyse and reflect on their training and translating. At the same time, the students of SG 3 and 4 highly evaluated the teacher feedback as the functional guidelines for their translation improvement. It is interesting to note, that the students of SG 1 preferred the feedback with low cognitive load, while the students of the other sample groups opted for medium or even high ones. This choice can be explained with more engaging nature of the task performed and strongly correlates with the results obtained by Adams, et al. [36] concerning the impact of implicit and explicit feedback types on learning outcomes and students' perception. All the respondents of SG 2-4 acknowledged that they had studied teacher feedback thoroughly because their next activities had been based on it. Almost $90 \%$ of the respondents stressed the positive impact of the teacher feedback on their translation skills development.

Taking into account some new practical findings of this research, we can provide the following guidelines for the efficient feedback introduction into the translation classroom. Firstly, any translation task performed by the students should be followed with some kind of qualitative feedback. As for junior students, it should be individual and teacher-generated. Teacher feedbacks should gradually develop from explicit mistake corrections accompanied with comments and explanations (feedback of low cognitive load) to simple mistakes location in the TT or even general comments how to improve it (feedback of high cognitive load) in the process of training. Secondly, teachers should urge their students to utilize the received feedback properly determining clearly the required form of their response. Thirdly, combination of some activities, directed at the deep analysis of the feedback content and its practical implementation, proved to be the most helpful at the initial stages of translation training. It can take the form of students' self-reflection reports containing their comments on the reasons and character of the made mistakes and ways to fix them now and prevent in the future followed with the obligatory correction of their TTs based on the received and analysed teacher feedback. We can assume here that self-report itself is not efficient enough to be used independently, while TT correction only may be successfully utilised at the next training stages, when the relevant skills of feedback analysis and application are already acquired by the students.

However, the conducted research has certain limitations, which may affect its outcome generalizability. Firstly, it worked pretty well for teaching abstract translation from English into Ukrainian to the second-year students with under-developed learning autonomy and self-regulation skills in the context of Ukrainian universities. Therefore, similar investigations can be conducted for other student populations, language combinations and types of translation or interpretation. 


\section{CONCLUSION}

The given research proved the importance of the student's active position in the feedback loop. The conducted survey verified the viability of the feedback loop designs involving different types of the students' response on the received teacher feedback. The response on the feedback in the form of the written selfreflection report preceding translation correction and resubmission proved empirically its higher effect compared to the self-reflection report or correction only, nothing to say about the students' passive studies of the teacher feedback and use of the obtained information for the improvement of their further translations. The students also recognised the positive impact of the teacher written individual feedback, and closely related its efficacy with its prospective practical application. The further investigation should concern the delivery and use of the teacher feedback regarding the students' individual learning styles and self-regulation skills.

\section{REFERENCES}

[1] C. J. Everhard, "The Assessment-Autonomy Relationship," in C. J. Everhard and L. Murphy, Eds., Assessment and Autonomy in Language Learning. Palgrave Macmillan, 2015, pp. 8-34.

[2] H. J. Kockaert and W. Segers, "Evaluation of legal translations: PIE method (Preselected Items Evaluation)," Journal of Specialised Translation, vol. 27, pp. 148-163, 2017.

[3] D. Boud and R. Soler, "Sustainable Assessment Revisited," Assessment \& Evaluation in Higher Education, vol. 41, no. 3, pp. 400-413, 2016.

[4] R. Dann, "Assessment as learning: blurring the boundaries of assessment and learning for theory, policy and practice," Assessment in Education: Principles, Policy \& Practice, vol. 21, no. 2, pp. 149-166, 2014.

[5] D. Kelly, A Handbook for Translator Trainers: A Guide to Reflective Practice Translation. Manchester/Northampton: St. Jerome, 2005.

[6] D. R. Sadler, "Formative Assessment: Revisiting the Territory," Assessment in Education: Principles, Policy \& Practice, vol. 5, no. 1, pp. 77-84, 1998.

[7] V. J. Shute, "Focus on formative feedback," Review of Educational Research, vol. 78, no. 1, pp. 153-189, 2008

[8] T. M. Južnič, "Assessment Feedback in Translator Training: A Dual Perspective," in N. K. Pokorn and K. Koskinen, Eds., New Horizons in Translation Research and Education. Joensuu: University of Eastern Finland, 2013, pp. 75-99.

[9] H. M. Alfayyadh, "The Feedback Culture in Translation Education: a Comparative Exploration of Two Distinct University Translation Programs," Ph.D. Dissertation, Kent State University, USA, 2016.

[10] P. Pietrzak, "Towards Effective Feedback to Translation Students: Empowering through Group Revision and Evaluation," inTralinea, Special issue: Challenges in Translation Pedagogy, pp. 1-6, 2014. [Online]. Available: http://www.intralinea.org/specials/article/2095.

[11] J. Lee, "Feedback on feedback: Guiding student interpreter performance," The International Journal for Translation \& Interpreting Research, vol. 10, no. 1, pp. 152-170, 2018.

[12] W. Neunzig and H. Tanqeiro, "Teacher Feedback in Online Education for Trainee Translators," Meta, vol. 50, no. 4, 2005, doi: 10.7202/019873ar.

[13] K. Washbourne, "Beyond error marking: written corrective feedback for a dialogic pedagogy in translator training," The Interpreter and Translator Trainer, vol. 8, no. 2, pp. 240-256, 2014.

[14] M. A. Erdoğan, "Exploring the Effectiveness of Using Audio-Recorded Feedback on Students' Writing Assignments," The fourth International scientific and practical Internet conference "Foreign Language in Professional Training of Specialists: Issues and Strategies", 2020, pp. 99-105.

[15] M. M. Bless, "Impact of Audio Feedback Technology of Writing Instruction," Master's Thesis, Southern Connecticut State University, USA, 2017.

[16] F. V. Lim and J. Phua, "Teaching Writing with Language Feedback," Technology, Computers and Composition, vol. 54, pp. 1-13, 2019.

[17] J. Boereboom and K. Moore, "Blurring the Boundaries: The impact of Formative Feedback on Summative Assessment," Research and Development in Higher Education in a Globalized World, vol. 37, pp. 39-47, 2014.

[18] D. Tsagari, "Interface between feedback, assessment and distance learning written assignments," Research Papers in Language Teaching and Learning, vol. 10, no. 1, pp. 72-99, 2019.

[19] O. Synekop, "Webquest as Technology of Differentiated ESP Instruction at University Level," Journal of Teaching English for Specific and Academic Purposes, vol. 8, no. 1, Special Issue, pp. 43-52, 2020.

[20] A. M. Selvaraj and H. Azman, "Reframing the effectiveness of feedback in improving teaching and learning achievement," International Journal of Evaluation and Research in Education (IJERE), vol. 9, no. 4, pp. 1055-1062, 2020.

[21] A. Khoram, A. D. Bazvand, and J. S. Sarhad, "Error Feedback in Second Language Speaking: Investigating the Impact of Modalities of Error Feedback on Intermediate EFL Students' Speaking Ability," Eurasian Journal of Applied Linguistics, vol. 6, no. 1, pp. 63-80, 2020.

[22] A. A. Zarei and H. Rezadoust, "The Effects of Scaffolded and Unscaffolded Feedback on Speaking Anxiety and Self-efficacy," Journal of Modern Research in English Language Studies, vol. 7, no. 4, pp. 111-132, 2020.

[23] J. Yang and C. B. Reiss, "Using Computer Assisted Translation tools' Translation Quality Assessment functionalities to assess students' translations," The Language Scholar, vol. 1, pp. 1-17, 2017. 
[24] L. Lim, "Examining students' perceptions of computer-assisted interpreter training," The Interpreter and Translator Trainer, vol. 7, no. 1, pp. 71-89, 2013.

[25] M. Barker and M. Pinard, "Closing the feedback loop? Iterative feedback between tutor and student in coursework assessments," Assessment \& Evaluation in Higher Education, vol. 39, no. 8, pp. 899-915, 2014.

[26] D. R. Sadler, "Beyond feedback: Developing student capability in complex appraisal," Assessment \& Evaluation in Higher Education, vol. 35, pp. 535-550, 2010.

[27] M. Yang and D. Carless, "The feedback triangle and the enhancement of dialogic feedback processes," Teaching in Higher Education, vol. 18, no. 3, pp. 285-297, 2013.

[28] J. Hattie and H. Timperley, "The power of feedback," Review of Educational Research, vol. 77, no. 1, pp. 81-112, 2007.

[29] R. Lyster and L. Ranta, "Corrective feedback and learner uptake," Studies in Second Language Acquisition, vol. 19, pp. 37-66, 1997.

[30] D. Brown, "The type and linguistic foci of oral corrective feedback in the L2 classroom: A meta-analysis," Language teaching research, vol. 20, no. 4, pp. 436-458, 2016.

[31] S. Ahmadi-Azad, "The Effect of Coded and Uncoded Written Corrective Feedback Types on Iranian EFL Learners' Writing Accuracy," Theory and Practice in Language Studies, vol. 4, no. 5, pp. 1001-1007, 2014.

[32] R. Ellis, Y. Sheen, M. Murakami, and H. Takashima, "The effects of focused and unfocused written corrective feedback in English as a foreign language context," System, vol. 36, no. 3, pp. 353-371, 2008.

[33] C. G. Van Beuningen, "Corrective feedback in L2 writing: Theoretical perspectives, empirical insights, and future directions," International Journal of English Studies, vol. 10, no. 2, pp. 1-27, 2010.

[34] S. Värlander, "The role of students' emotions in formal feedback situations," Teaching in Higher Education, vol. 3, no. 2 , pp. 145-156, 2008.

[35] K. O. Shevelko, "The methodology of teaching abstract and gist translation of publicistic texts in English to senior pupils of secondary schools specializing in foreign languages," Ph.D. Dissertation, Kyiv National Linguistic University, Kyiv, Ukraine, 2016.

[36] R. Adams, A. M. Nuevo, and T. Egi "Explicit and Implicit Feedback, Modified Output, and SLA: Does Explicit and Implicit Feedback Promote Learning and Learner-Learner Interactions?" The Modern Language Journal, vol. 95, no. s1, pp. 42-63, 2011. 